\title{
Neurolinguistic aspects of metaphor theory
}

Georgia Andreou and Ioannis Galantomos

Department of Special Education, University of Thessaly, Greece

https://doi.org/10.36505/ExLing-2008/02/0005/000064

\begin{abstract}
The goal of this paper is to examine the neural aspect of metaphor. The neural theory of language stems from the cognitive linguistic research and is an effort to comprehend the way neural circuits affect and shape language and thought. Within this framework it is suggested that metaphor serves as the medium through which cultural, abstract and theoretical concepts are acquired. On the other hand, it is believed that conceptual metaphors are ensembles of neurons located in different parts of the brain and connected by larger neural groups.
\end{abstract}

Key words: cognitive linguistics, conceptual metaphor, neural circuits, neural mappings

\section{Introduction}

The traditional view holds that metaphor is a figure of speech, a property of words, which is used mainly for rhetorical and artistic purposes (Kövecses 2002). Lakoff and Johnson (1980/2003) in their seminal work "Metaphors we live by", based on a big volume of empirical data, challenged those well established beliefs and pointed at the pervasiveness of metaphor in everyday language. Furthermore, they claimed that the human conceptual system is metaphorical in nature and that abstract thinking relies on metaphor.

In the cognitive linguistic approach, metaphor is a complex phenomenon. In particular, it is suggested that metaphor is conceptual, linguistic, bodily, socio-cultural and neural.

Metaphor is a property of concepts and it refers to the understanding of one less physical/abstract domain (i.e. concept) in terms of a more concrete one. This is called a conceptual metaphor and it consists of a source and a target domain. Thus, in the conceptual metaphor LOVE IS A JOURNEY (conceptual metaphors by convention are written in small capitals), love is the target domain, whereas journey is the source domain. Conceptual metaphors become explicit in language through metaphorical linguistic expressions. An example of the above mentioned conceptual metaphor is the linguistic expression "we are at a crossroads".

Between the source and the target domain there are systematic correspondences which are called mappings. For instance, in the metaphor LOVE IS A JOURNEY the travellers correspond to the lovers. Conceptual metaphors are grounded in embodied experience, that is the way human body operates exerts an influence on the final choice of a certain source

ExLing 2008: Proceedings of 2nd Tutorial and Research Workshop on Experimental Linguistics, 25-27 August 2008, Athens, Greece 
domain to go with a particular target domain. Also, metaphors reflect cultural patterns. In other words, entrenched cultural models affect human understanding of certain concepts.

The neural basis of metaphor is one of the current findings of cognitive researchers. The major assumption is that metaphors are comprehended through the activation of particular neural combinations that are located in the sensorimotor system and in higher cortical areas (Kövecses 2005).

Earlier results, outside the cognitive linguistic research, suggested the contribution of the right hemisphere $(\mathrm{RH})$ to the comprehension of metaphor (e.g. Burgess and Chiarello 1996). Nevertheless, current findings (e.g. Lee and Dapretto 2006) challenge the selective role of the RH and argue against its specialization in metaphor processing.

\section{The neural theory of metaphor}

The neural theory of metaphor and by extension of language is a project that is taking place at the University of California at Berkeley aiming at understanding the role of neural circuits in language and thought (Gibbs 2006).

Within this framework, three models were developed. The first one was proposed by Regier (1996) who designed a neural model for acquiring spatial terms in various languages. Regier used topographical maps of the visual field, orientation-sensitive cell systems to compute the orientational aspects of spatial concepts that are related to bodily orientation and centersensitive receptor fields in order to characterize concepts, such as contact and distance. Regier's model showed very good results in learning these spatial terms.

The second model is Narayanan's (1997) neural theory of metaphor. This project laid an emphasis on metaphorical inferences about events and the verbal category of aspect. Narayanan used computational methods for neural modelling and showed that conceptual metaphors are neuronal combinations located in different parts of the brain and connected by the functions of neural circuits. These combinations are the source and the target domain and the neural circuits are the mappings. Under this perspective, mappings are not anymore simple correspondences between two interrelated domains but are neural mappings that constitute the neural mechanism, which connects the sensorimotor system (where the source domain is located) and higher cortical areas (where the target domain is located).

A last model was developed by Bailey (1998) who attempted to explain the acquisition of hand motion verbs (e.g. push, grasp, wave). Bailey concluded that human body serves as the basis for defining the meanings each hand motion verb has in world's languages. In other words, he 
presented proof for the key-role of the sensorimotor system for distinguishing among the semantics of the hand motion verbs.

Among these neural models the most important is the second one, namely Narayanan's neural theory of metaphor which explains adequately the findings of two other advances in cognitive linguistics. The first advance is Grady's (1997) primary metaphor theory which is an attempt to explain the partial nature of metaphorical mappings. For this reason, Grady introduced the notion of primary metaphor in order to address the weakness of the standard metaphor theory to justify why some elements are mapped onto one domain and not others. Primary metaphors create complex metaphor(s) and stand at the higher level of abstraction.

The acquisition of these primary metaphors led Johnson (1997) to conduct an experiment in order to test when children learn the metaphorical sense of the verb see as in the sentence "I see what you mean". Johnson found that the literal meaning of this verb was acquired first. Then, a conflation period followed where both literal and metaphorical meanings were present and active and finally pure metaphorical cases were learned. Johnson's findings serve as the second advance.

The results of both Grady's and Johnson's experiments are best explained by Narayanan's neural theory of metaphor. The primary metaphors are learned on the basis of neural mappings that connect the source and the target domain and are active during the conflation period. New metaphors are learned through the establishment of new neural mappings and not the emergence or the creation of a copy machinery. One implication is that there is no need of overrides because metaphors are acquired when two experiences occur at once. If a neural mapping in the target domain leads to a contradiction then it will not be learned and it will be inhibited.

According to Lakoff and Johnson (1980/2003) metaphor in the neural theory of language has certain advantages. First, it explains the way primary metaphors are learned. Second, it explains the ubiquity of metaphors. Third, there is no need of overrides. Fourth, metaphors fit well with the other aspects of this theory. Fifth, it can explain the dynamic role and the use of metaphor in everyday language and finally it offers a computational model for the study of metaphor in discourse.

\section{Conclusions}

In sum, metaphor, within the neural theory of language, becomes a neural mechanism. An entailment of this view is that the most abstract products of the human conceptual system, namely the primary metaphors, are acquired automatically and unconsciously during the period of conflation. People have no choice in avoiding this because of the stable and constant way neural mappings operate and connect sensorimotor system and higher 
cortical areas of the brain each time they attempt to understand abstract concepts in metaphorical terms.

\section{References}

Bailey, D. 1998. Getting a Grip: A Computational Model of the Acquisition of Verb Semantics for Hand Actions. Unpublished doctoral dissertation. University of California, Berkeley, USA.

Burgess, C. and Chiarello, C. 1996. Neurocognitive Mechanisms Underlying Metaphor Comprehension and Other Figurative Language. Metaphor and Symbolic Activity 11, 67-84.

Gibbs, R.W. Jr. 2006. Embodiment and Cognitive Science. Cambridge: CUP.

Grady, J. 1997. Foundations of Meaning: Primary meanings and primary senses. Unpublished doctoral dissertation. University of California, Berkeley, USA.

Johnson, C. 1997. Metaphor vs conflation in the acquisition of polysemy. The case of SEE. In Hiraga, M.K., Sinha, C. and Wilcox, S. (eds.), Cultural, typological and psychological issues in cognitive linguistics. Current Issues in Linguistic Theory, vol. 152, 155-169. Amsterdam, John Benjamins.

Kövecses, Z. 2002. Metaphor. A Practical Introduction. Oxford: OUP.

Kövecses, Z. 2005. Metaphor in Culture. Universality and Variation. Cambridge: CUP.

Lakoff, G. and Johnson, M. 1980/2003. Metaphors we live by. Chicago and London: The University of Chicago Press.

Lee, S.L. and Dapretto, M. 2006. Metaphorical vs. literal word meanings: fMRI evidence against a selective role of the riht hemisphere. NeuroImage 29, 536-544.

Narayanan, S. 1997. Moving Right Along: A Computational Model of Metaphoric Reasoning about Events. Unpublished doctoral dissertation. University of California, Berkeley, USA.

Regier, T. 1996. The Human Semantic Potential. Chicago: The University of Chicago Press. 